\title{
Penile Cancer by AJCC v7 Stage
}

National Cancer Institute

\section{Source}

National Cancer Institute. Penile Cancer by A/CC v7 Stage. NCI Thesaurus. Code C91234.

A term that refers to the staging of penile carcinoma according to the American Joint Committee on Cancer, 7th edition. 